\title{
COMET Experiment searching for muon to electron conversiton
}

\author{
Y. Nakazawa ${ }^{* A}$ Y. Kuno ${ }^{A}$, A. Sato ${ }^{A}$, H. Sakamoto ${ }^{A}$, H. Yoshida ${ }^{A}$, Z. Jie ${ }^{B}$, C. WU $\mathbf{W}^{B, C}$, H. \\ Katayama $^{A}$, T.S. Wong ${ }^{A}$, the COMET Collaboration \\ ${ }^{A}$ Department of Physics, Osaka University \\ ${ }^{B}$ Institute of High Energy Physics, China \\ ${ }^{C}$ NanJing University, China \\ E-mail: घ-nakazawadkuno-q.phys.sci.osaka-u.ac.jp
}

The COMET is one of experiments that search for new physics beyond the Standard Model. It is dedicated to searching for charged lepton flavor violation processes with muon to electron in a muonic atom $\left(\mu^{-} N \rightarrow e^{-} N\right)$. A staging approach is planed for the COMET experiment. The COMET Phase-I plans to achieve a signal sensitivity of $3 \times 10^{-15}$ in 2017 followed by the COMET Phase-II with the sensitivity of $10^{-17}$ in 2020.[1]

In this paper, I introduce status of the COMET Phase-I, which consists of a proton beam line, a muon beam section and a detector section. A pion capture system will be installed at the end of the proton beam line to provide a very intense muon beam of $1 \times 10^{9} \mu / \mathrm{sec}$. The pion capture system is currently under construction at J-PARC. The detector section has a muon stopping target surrounded by a Cylindrical Drift Chamber (CDC).

Flavor Physics \& CP Violation 2015

May 25-29, 2015

Nagoya, Japan

${ }^{*}$ Speaker. 


\section{Introduction}

The COMET is dedicated to searching for charged Lepton Flavor Violation (cLFV) processes with muon to electron conversion in a muonic atom $(\mu N \rightarrow e N)$. A staging approach is planed for the COMET experiment. The COMET Phase-I plans to achieve a signal sensitivity of $3 \times 10^{-15}$ in 2017 followed by the COMET Phase-II with the sensitivity of $10^{-17}$ in 2020.

Currently COMET Group is preparing the proton beam line, a muon beam section and a detector section for the COMET Phase-I (Fig1). A detector design is fixed by performance tests and constructing a Cylindrical Drift Chamber (CDC) starts.

\section{Physics}

The COMET aims at the observation of the neutrinoless muon to electron conversion. Muons are captured by the aluminum stopping target. Then the signal from the $\mu-e$ conversion is a mono-energetic electron by the following energy calculation.

$$
E_{e}=m_{\mu}-B_{\mathrm{Al}}-R_{\mathrm{Al}} \sim 104.9 \mathrm{MeV}
$$

( $E_{e}$ is an energy of signal electron. $m_{\mu}$ is muon mass. $B_{\mathrm{Al}}$ is binding energy of $\mathrm{Al} . R_{\mathrm{Al}}$ is atomic nuclear recoil effect.) In the COMET Phase-I, these electrons emitted from muon decays are detected by the CDC. The CDC surrounds the aluminum target, so background events from this target is very serious problem. Main background is an electron emitted from a muon's three body decay with two neutrinos in an $\mathrm{Al}$ atomic orbit (Decay in Orbit ; DIO).

$$
\mu^{-} \rightarrow e^{-} v_{\mu} \overline{v_{\mathrm{e}}}
$$

Normally a muon emits an electron which has less than $\sim 53 \mathrm{MeV}$ with tree body decay. However, due to an $\mathrm{Al}$ atomic nuclear recoil, momentum spectrum of DIO electrons has a long tail near the signal region. So the CDC which achieves a high momentum resolution is required(Fig2).[2]

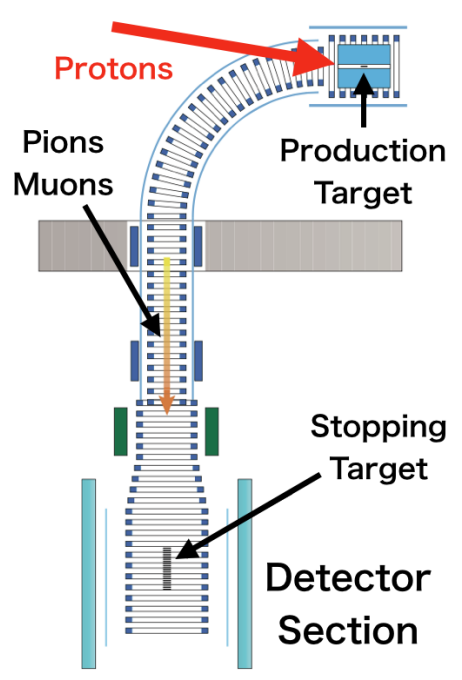

Figure 1: Schematic of COMET Phase-I Proton beam from a main ring of J-PRAC hits the production target and produces pions. Pions are transported while there emit muons in decay. Muons are captured at stopping target and decay to electrons. Electrons emitted from the target are detected by the CDC

\section{Cylindrical Drift Chamber}

A momentum resolution of the CDC must be less than $200 \mathrm{keV} / \mathrm{c}$ for achieving a target sensitivity. So an electron beam test at Tohoku University and a gain test at Osaka University are done. In China IHEP Group tested DAQ connection. From these tests results, the detector group is finalizing the CDC design and DAQ system. We start constructing the CDC and DAQ system. 


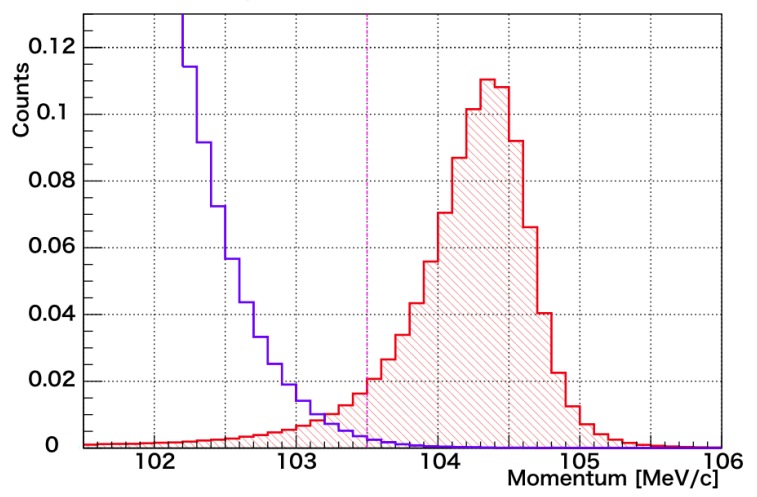

Figure 2: Momentum Spectrum

Red graph shows momentum spectrum of electrons emitted by $\mu-e$ conversion. Blue graph shows one of DIO electrons. DIO spectrum seeps into the signal region.

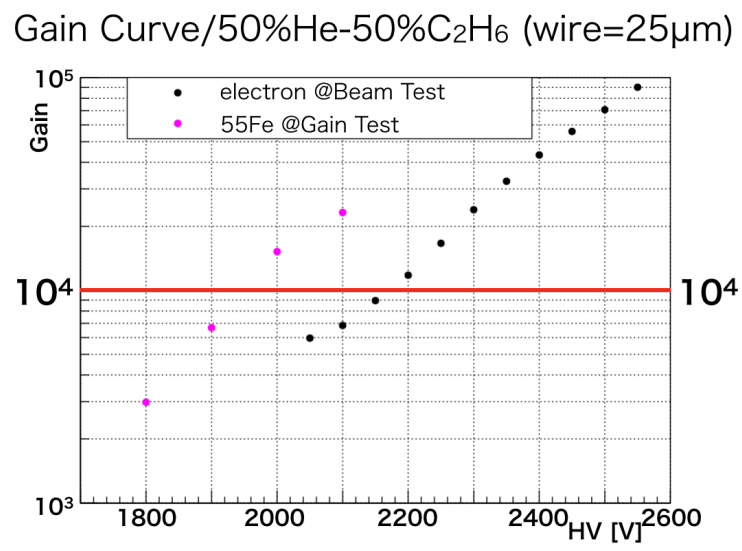

Gain Curve/50\%He-50\% $\mathrm{C}_{2} \mathrm{H}_{6}$ (wire=30 $\mu \mathrm{m}$ )

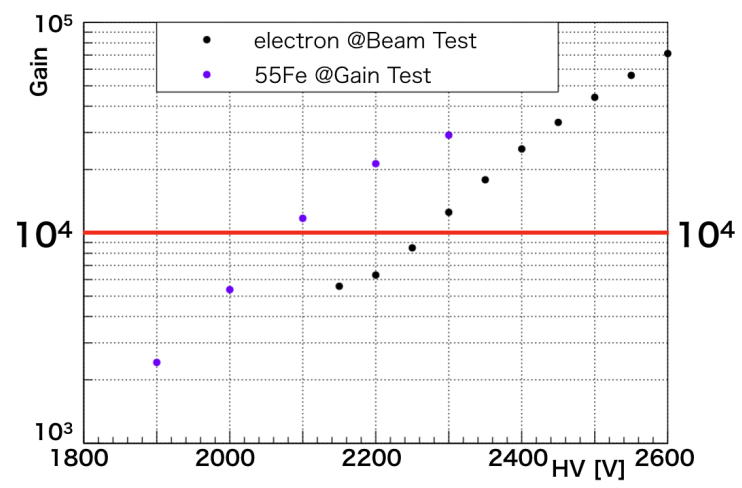

Figure 3: Momentum Spectrum

Upper graph shows wire diameter of $25 \mu \mathrm{m}$. Lower graph shows wire diameter of $30 \mu \mathrm{m}$. These graph compare between tohoku beam test and gain test. 


\section{Gain Test}

According to a previous design, a candidate of field wire is $\phi 80 \mu \mathrm{m} \mathrm{Al}$ wire and sense wire is $\phi 25 \mu \mathrm{m}$ or $\phi 80 \mu \mathrm{m} \mathrm{Au}-\mathrm{W}$ wire. Wire diameter is decided by Electron Beam Test and Gain Test result(Fig 3).[3]

\subsection{Field Wire}

To avoid an aging effects, a surface $\mathrm{E}$ field on field wire need be less than $20 \mathrm{kV} / \mathrm{cm}$ and this corresponds to applied voltage on sense wires less than $1700 \mathrm{~V}$. We want to operate the CDC with the gas gain of $10^{4} \sim 10^{5}$ for stable working. To satisfy this condition, applied voltage should be higher than $\sim 2000 \mathrm{~V}$. And the beam test result shows that the highest efficiency is $\sim 96 \%$ which is at $2350 \mathrm{~V}$. Therefore field wire is chosen to be $\phi 126 \mu \mathrm{m}$ to reduce the surface E field.[4]

\subsection{Sense Wire}

For low energy electron search, the momentum resolution of the CDC is dominated by multiple scattering effects. Therefore the CDC must be a low-mass detector. According to two tests, both sense wire candidates can work stability. So the diameter of sense wire is fixed to $\phi 25 \mu \mathrm{m}$.

\section{DAQ connection Test}

In this test with a setup of Fig4, there are three evaluation items.

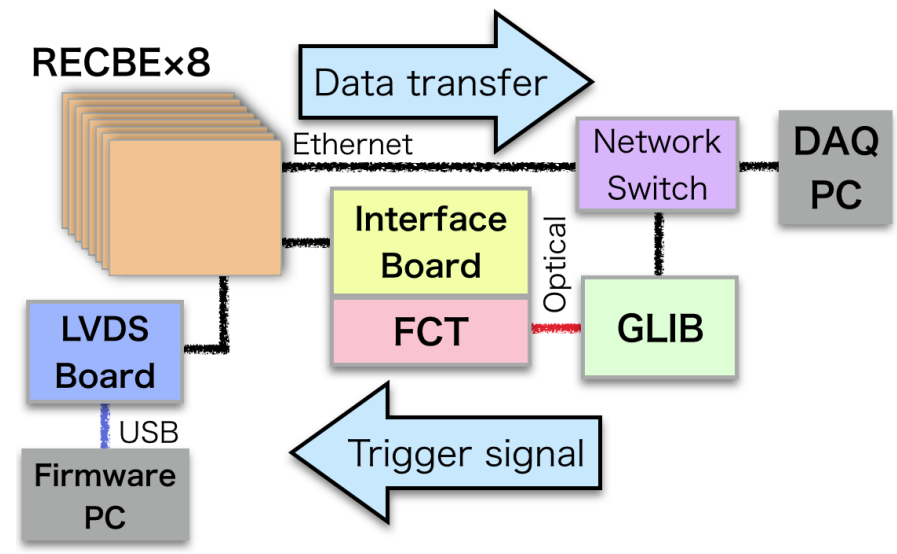

Figure 4: Setup at the DAQ connection test

\section{(1)Operation test of the new RECBEs for the COMET}

In the COMET Phase-I, the RECBE which is an electric circuit developed by Belle-II group for data acquisition is used the CDC's DAQ system. The RECBE for the Belle-II can not work for the CDC. So IHEP group in China remakes the RECBE and removes unnecessary parts. In this test, we could get a data of waveform made by a function generator from new RECBEs reproduced for COMET at IHEP with using the setup of Fig4. 


\section{(2)Downloading a firmware through ethernet cable}

In this test, it is checked that a firmware is downloaded by remote operation with ethernet cables at beam time. The result is that downloading a firmware through ethernet cables (Cat 7) was successful.

\section{(3)RECBE-FCT-GLIB communication}

GLIB which is trigger decision and timing control electronics connects the CDC with trigger detector. And FCT connects RECBEs with GLIB. So we have to check these communication to send a trigger to RECBEs through GLIB and FCT. Three following tests were successful.

- Sending a trigger number from GLIB via FCT and recording this number to DAQ PC through SiTCP of RECBEs

- Sending busy signals from RECBEs to GLIB via FCT to stop the trigger sending

- Sending a $40 \mathrm{MHz}$ clock from GLIB via FCT to RECBEs and synchronize among RECBEs

\section{CDC Construction}

From these result, the CDC design is fixed. Thereby detector group stars constructing the real CDC at FUJI experimental building in KEK (Fig5). And a mass-production of RECBEs for the CDC is about to start at IHEP.

\section{Summary \& Prospect}

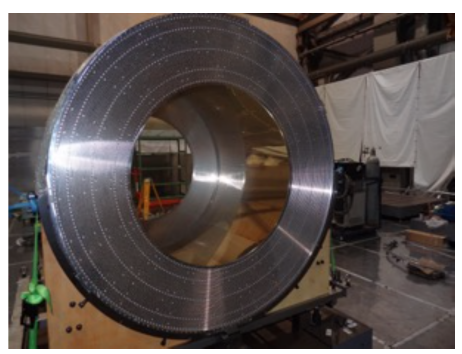

The COMET Phase-I is searching for the electron emitted from $\mu^{-} N \rightarrow e^{-} N$ with the sensitivity of $10^{-15}$ to discovery cLFV process. For achieving this purpose, a construction of the CDC and DAQ system starts. A new prototype of the CDC with new design was constructed, so the final performance test will be done in this summer. Then residual parameters which have not decided yet will be examined.

\section{References}

[1] COMET collaboration, Technical Design Report

[2] A. Czarnecki et al., Muon decay in orbit: Spectrum of High-energy electrons, Phys. Rev. D 84, 013006 (2011)

[3] Y. Nakazawa et al., COMET Phase-I CDC Prototype Beamtest 1, 70th JPS meeting at Waseda University, Mar. 212015

[4] T.S Wong et al., Development of Cylindrical Drift Chamber for COMET Phase-I, FPCP 2015 at Nagoya University 